The International Journal Of Engineering And Science (IJES)

|| Volume || 6 || Issue || 3 || Pages || PP 14-20 || 2017 ||

ISSN (e): $2319-1813 \operatorname{ISSN}(p): 2319-1805$

THE IJES

\title{
Numerical Calculation of Solid-Liquid two-Phase Flow Inside a Small Sewage Pump
}

\author{
Yu-Liang Zhang ${ }^{1}$,Xiao-Jun Yang $^{1}$, Yan-Juan Zhao ${ }^{2}$ \\ $\left({ }^{l}\right.$ College of Mechanical Engineering, Quzhou University, Quzhou 324000, China; \\ ${ }^{2}$ College of Information Engineering, Quzhou College of Technology, Quzhou 324000, China)
}

\begin{abstract}
-
Based on a mixture multiphase flow model,theRNG $k$-Eturbulencemodelandfrozen rotor method were used to perform a numerical simulation of steady flow in the internal flow field of a sewage pump that transports solid and liquid phase flows. Resultsof the study indicate that the degree of wear on the front and the back of the blade suction surface from different densities of solid particles shows a completely opposite influencing trend. With the increase of delivered solid-phase density, the isobaric equilibrium position moves to the leading edge point of the blade, but the solid-phase isoconcentration point on the blade pressure surface and suction surface basically remains unchanged. The difference between hydraulic lift and water lift indelivering solid-and liquid-phase flows shows a rising trend with the increase of working flow.
\end{abstract}

Keywords:sewage pump, numerical simulation, frozen rotor method, flow characteristics

\section{INTRODUCTION}

Sewage pumpsconsist of both solid- and liquid-phase flow pumps and are key equipment for urban channel cleanout[1 11]. Many scholars at home and abroad have carried out in-depth and meticulous research on sewage pumps and have achieved productive results. However, with the wide application of the solid-liquid pump, the shortcomings and deficiencies in the actual use were gradually exposed,such as low efficiency and excessively wornwet parts. Meanwhile, due to the complexity of two-phase flow, the theoretical research and design in this field is not mature. As the design of most existing products was based on the experiment, the designed pump performance could not be guaranteed. Therefore, studying the internal flow of the pump to provide a theoretical basis for realizingahigh-performingand stable-operating pump design is needed. The presentstudyaims to conductanumerical simulation and analysis on solid-liquid two-phase flow pump to analyze the impact of the density of solid-phase particles on sewage pump performance, including the hydraulic performance of sewage pump and wear degree of wet parts. Through the analysis ofthe internal pressure and dynamic deposition distribution of particles on the surface of the solid, the basic properties of sewage pump and theoretical foundation for further design improvement could be predicted.

2 Physical model and solving method

\section{PHYSICAL MODEL}

The model was a medium specific speed centrifugal pump. Its basic performance parameters were as follows: flow rate $Q=50 \mathrm{~m}^{3} / \mathrm{h}$, head $H=20.54 \mathrm{~m}$, velocity $n=2900 \mathrm{r} / \mathrm{min}$, shaft power $P_{a}=3.54 \mathrm{~kW}$, efficiency $\eta=79.5 \%$, andnet positive suction head $N P S H_{\mathrm{C}}=2.59 \mathrm{~m}$. The main dimensions of the pumpwereas follows: inlet diameter $D_{j}=76 \mathrm{~mm}$, hub diameter $d_{h}=0 \mathrm{~mm}$, impeller outlet diameter $D_{2}=137 \mathrm{~mm}$, impeller outlet width $b_{2}=14 \mathrm{~mm}$, blade outlet angle $\beta_{2}=30^{\circ}$.

\subsection{Computational domain}

The appropriate extension of the straight-tube suction chamber was made to eliminate the influence of the given inlet speed. Anappropriateextension of the pump outlet was also carried outto better calculate convergence. As shown in Fig. 1,Tetrahedralmesheswere adopted in the impeller rotation region and volute static region, with mesh numbers 435,530 and 597,127 respectively. Hexahedral mesheswere used in the suction chamber and outlet extension, with mesh numbersof 152,827 and 54,944 respectively. The total mesh number of the overall computational domain was $1,240,428$. 


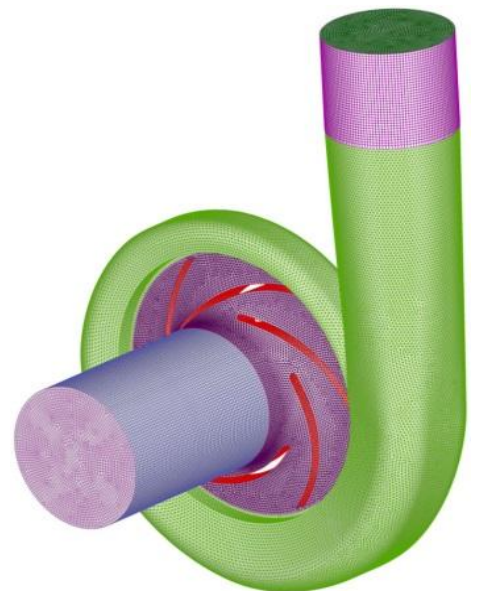

Fig.1 Computational mesh

\subsection{Solved equations}

According totheBoussinesq approximation,

$$
-\rho \overline{u_{i} u_{j}^{\prime}}=\mu_{t}\left(\frac{\partial \bar{u}_{i}}{\partial x_{j}}+\frac{\partial \bar{u}_{j}}{\partial x_{i}}\right)-\frac{2}{3}\left(\rho k+\mu_{t} \frac{\partial \bar{u}_{i}}{\partial x_{i}}\right) \delta_{i j}(1)
$$

Where $\mu_{t}$ refers to turbulent viscosity, which is the function coefficient of turbulent kinetic energy $k$ and turbulent dissipation rate $\varepsilon$.

Unsteady turbulent flow calculation used in the present study usedthe RNG $k-\varepsilon$ two-equation model to close the mean Reynolds stress. RNG $k-\varepsilon$ turbulent model, which considers the rotation and swirling flow in the mean flow, can better process the flow with high strain rate and great streamline curvature. The RNG $k-\varepsilon$ turbulentequations are as follows:

$$
\begin{aligned}
\rho \frac{\mathrm{d} k}{\mathrm{~d} t}=\frac{\partial}{\partial x_{j}}\left(\alpha_{k} \mu_{\mathrm{eff}} \frac{\partial k}{\partial x_{j}}\right)+2 \mu_{t} \bar{S}_{i j} \frac{\partial \bar{u}_{i}}{\partial x_{j}}-\rho \varepsilon(2) \\
\qquad \frac{\mathrm{d} \varepsilon}{\mathrm{d} t}=\frac{\partial}{\partial x_{j}}\left(\alpha_{\varepsilon} \mu_{\mathrm{eff}} \frac{\partial \varepsilon}{\partial x_{j}}\right)+2 C_{1 \varepsilon} \frac{\varepsilon}{k} v_{t} \overline{S_{i j}} \frac{\partial \bar{u}_{i}}{\partial x_{j}}-C_{2 \varepsilon} \rho \frac{\varepsilon^{2}}{k}-R(3)
\end{aligned}
$$

where $\overline{S_{i j}}=\frac{1}{2}\left(\frac{\partial \bar{u}_{i}}{\partial x_{j}}+\frac{\partial \bar{u}_{j}}{\partial x_{i}}\right) ; \mu_{\mathrm{eff}}=\mu+\mu_{t} ; \mu_{t}=C_{\mu} \frac{k^{2}}{\varepsilon} \overline{S_{i j}}$ refers to the strain rate tensor, and $R$ refers to $\varepsilon$ additional source terms in the equation, representing the effect of mean strain rate $\varepsilon$.Itisexpressed as follows:

$$
R=\frac{C_{\mu} \eta^{3}\left(1-\eta / \eta_{0}\right)}{1+\beta \eta^{3}} \frac{\varepsilon^{2}}{k}, \eta=S k / \varepsilon
$$

The model parameters in the above equations are $C_{\mu}=0.0845, C_{1 \varepsilon}=1.42, C_{2 \varepsilon}=1.68, \alpha_{k}=1.0, \alpha_{\varepsilon}=0.769, \beta=0.012$, and $\eta_{0}=4.38$.

\subsection{Multiphase flow model}

The general form of the ASMM is as follows, i.e., continuity equation,

$$
\frac{\partial}{\partial t}\left(\rho_{m}\right)+\frac{\partial}{\partial x_{i}}\left(\rho_{m} \boldsymbol{u}_{m, i}\right)=0
$$

Where $\rho_{m}$ is the density of the mixture, and $\boldsymbol{u}_{m}$ is the mass average velocity. The momentum equation is

$$
\begin{aligned}
\frac{\partial}{\partial t}\left(\rho_{m} u_{m, j}\right) \frac{\partial}{\partial x_{i}}\left(\rho_{m} u_{m, i} u_{m, j}\right) & =-\frac{\partial p}{\partial x_{j}}+\frac{\partial}{\partial x_{i}} \times \mu_{m}\left(\frac{\partial u_{m, i}}{\partial x_{j}}+\frac{\partial u_{m, j}}{\partial x_{i}}\right) \\
+ & \rho_{m} g_{j}+\boldsymbol{F}_{j}+\frac{\partial}{\partial x_{i}}\left(\sum_{k=1}^{n} \varphi_{k} \rho_{k} u_{D, k, i} u_{D, k, j}\right)
\end{aligned}
$$


Wherepis the pressure, $\mu_{m}$ is the effective viscosity of the mixture, $g$ is the gravitational acceleration, $\boldsymbol{F}$ is the volume force, $\varphi$ is the volume fraction, and $u_{D, k}$ is the drift velocity.

The volume fraction equation is

$$
\begin{aligned}
& \frac{\partial}{\partial t}\left(\varphi_{s} \rho_{s}\right)+\frac{\partial}{\partial x_{i}}\left(\varphi_{s} \rho_{s} u_{m, i}\right)=-\frac{\partial}{\partial x_{i}}\left(\varphi_{s} \rho_{s} u_{D, s, i}\right)(7) \\
& \rho_{m}=\sum_{k=1}^{n} \varphi_{k} \rho_{k} \quad \mu_{m}=\sum_{k=1}^{n} \varphi_{k} \mu_{k} \quad \boldsymbol{u}_{m}=\left(\sum_{k=1}^{n} \varphi_{k} \rho_{k} \boldsymbol{u}_{k}\right) / \rho_{m}
\end{aligned}
$$

In this study, the calculated medium is a solid-liquid two-phase flow, where $n=2$. If a steady flow calculation is to be conducted, the phase of the local change rate will no longer exist in the above equation.

\subsection{Solution settings}

The dynamic and static coupling between rotor and stator in numerical calculation was achieved by means of the frozen rotor method. Velocity inlet and free output were respectively used as the boundary conditions of the inlet and outlet. Axial velocity of the pump inlet was calculated directly according to the given flow conditions and pump inlet diameter. Turbulent kinetic energy and turbulent dissipation rate under the corresponding flow were calculated based on axial velocity and pump inlet diameter. No-slip boundary condition was applied on all solid walls considering viscosity, and standard wall function was used in near-wall low Reynolds number region to deal with the problem from the high Reynolds number turbulence model. The coupling between velocity and pressure was calculated by using SIMPLE algorithm. Solid particles were uniform spherical particles with unchanged physical properties.

\subsection{Computational scheme}

Under a designed flow rate $\left(Q=50 \mathrm{~m}^{3} / \mathrm{h}\right)$ with $10 \%$ solid-phase concentration and $0.10 \mathrm{mmparticle}$ diameter, numerical calculation was conductedfora solid-liquid two-phase flow field under five conditions when the particledensitieswere $500,1000,1500,2000$, and $2500 \mathrm{~kg} / \mathrm{m}^{3}$. Numerical calculation of the solid-liquid two-phase flow field was carried outunder different working conditions:10\% solid-phase concentration, $0.10 \mathrm{mmparticle}$ diameter, and $2500 \mathrm{~kg} / \mathrm{m}^{3}$ particle density.

3Analysis of results

\section{TOTAL PRESSURE DISTRIBUTION OF BLADE UNDER DIFFERENT PARTICLE DENSITIES}

Fig. 2 shows the total pressure distribution on the blade pressure surface and blade suction surface in five different delivering densities of solid particles. Regardless of whether on pressure surface or suction surface, total pressure increased with the increase of the blade radius. In the case of the $500 \mathrm{~kg} / \mathrm{m}^{3}$ density of delivering solid particles, solid-phase density was smaller than the liquid-phase density. In most areas (relative length ofabout 0.96), the total pressure on the blade pressure surface was higher than that on the suction surface. The total pressure on the suction surface likewise started to be higher than that on the pressure surface. In other words, position 0.96I was an isobaric equilibrium point of the pressure surface and the suction surface. For the other four densities of delivering solid particles, the changing characteristic was similarlygeneral.The only difference was that the isobaric equilibrium pointswere in different positions. In the four cases of densities of delivering solid particles, i.e., $1000,1500,2000$, and $2500 \mathrm{~kg} / \mathrm{m}^{3}$, the relative positions of the isobaric equilibrium pointswere $0.84,0.75,0.72$, and 0.70 , respectively. Generally speaking, with the increase of delivering solid-phase density, the position of the isobaric equilibrium point was moving to the front edge point of the blade.
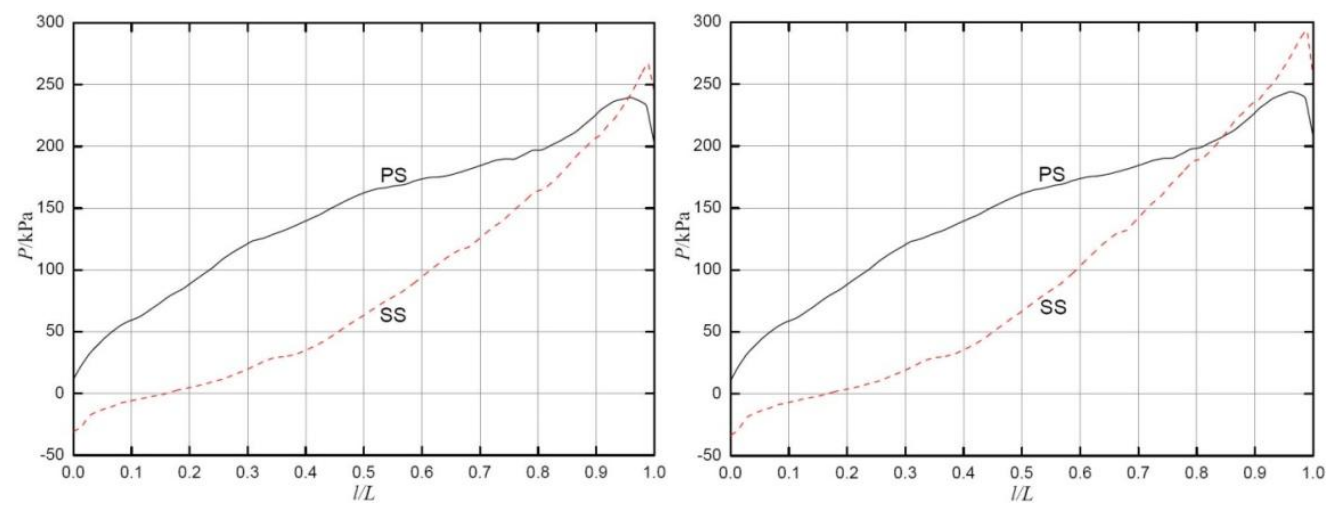

(a) $500($ b) 1000 


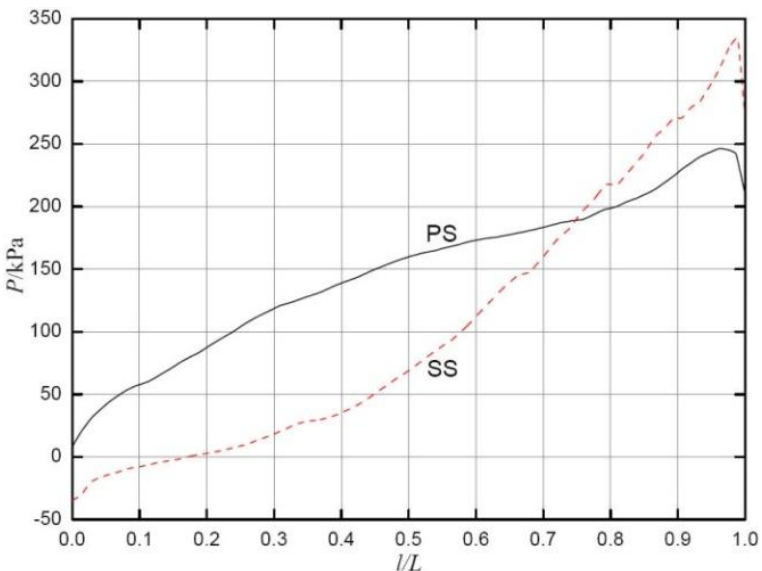

(c) 1500

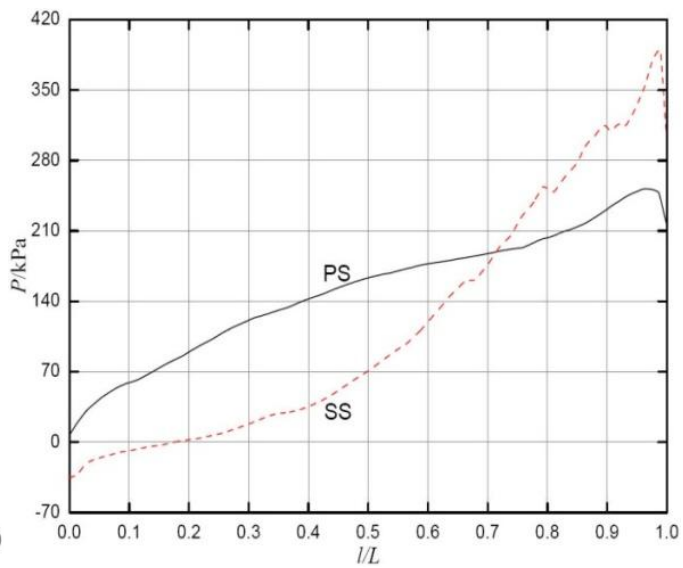

(d) 2000

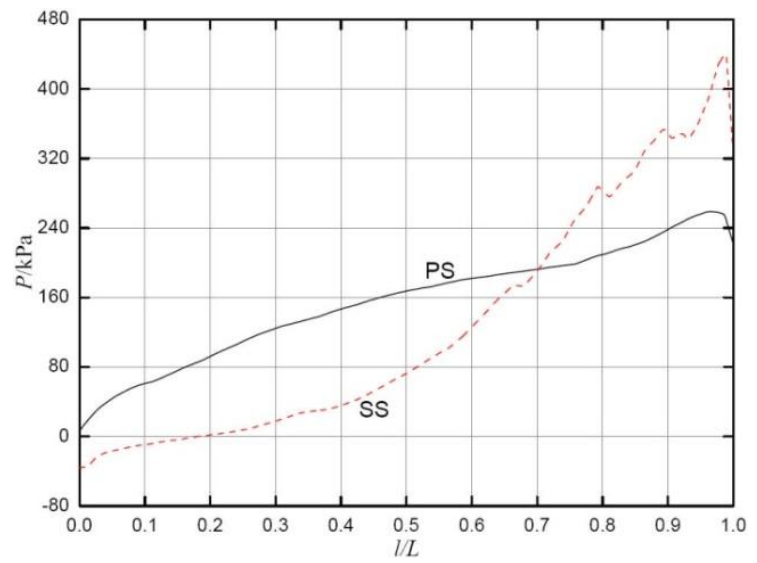

(e) 2500

Fig.2 Distribution and variation of total pressure on the blade under different particle densities $\left(\mathrm{kg} / \mathrm{m}^{3}\right)$

\subsection{Distribution of particle concentration of the blade under different particle densities}

Fig. 3 shows the distribution of solid-phase concentration on the blade pressure and suction surface under five densities of delivering solid particles. When the densities of delivering solid-phase particles were different, the difference in the concentration distributionswashighlysignificant. When the particle density $\left(500 \mathrm{~kg} / \mathrm{m}^{3}\right) \mathrm{was}$ smaller than the liquid-phase density (water) $\left(1000 \mathrm{~kg} / \mathrm{m}^{3}\right)$, the solid-phase concentration on the blade pressure surface was higher than that on the blade suction surface in most areas ofthe blade surface. This finding indicated that the blade pressure surface bore more serious wear. However, in the inlet of the blade, solid-phase concentration on the blade pressure surface was lower than that on the suction surface. Therefore, the suction surface bore more serious wear. Similarly, the position of the isoconcentration point (the point in the same radius on the blade pressure surface and the suction surface with same solid-phase concentration) was atapproximately0.11 (relative length). When particle density $\left(1000 \mathrm{~kg} / \mathrm{m}^{3}\right)$ was equal to liquid-phase density $\left(1000 \mathrm{~kg} / \mathrm{m}^{3}\right)$, the solid-phase concentration on the blade pressure surface was equal to that on the suction surface $(10 \%)$ within the full range of the blade surface.This finding indicated that the blade pressure surface and suction surface bore equal wear. When particle density $\left(1500,2000\right.$, and $\left.2500 \mathrm{~kg} / \mathrm{m}^{3}\right)$ were greater than liquid-phase density $\left(1000 \mathrm{~kg} / \mathrm{m}^{3}\right)$,solid-phase concentration on the blade suction surface was significantly higher than that on the pressure surface in most areas on the blade surface. This result indicated that the blade suction surface bore more serious wear. However, under the latter three conditions, the position of solid-phase isoconcentrationpointswasin $0.11,0.13$, and 0.13 (relative length). Specifically, the position of solid-phase isoconcentration point on the blade pressure and suction surface was almost unchanged with the increase of the density of delivering solid particles. This finding indicated that the degree of wear of the blade pressure surface and the suction surface did not change due to the change in particle density. 


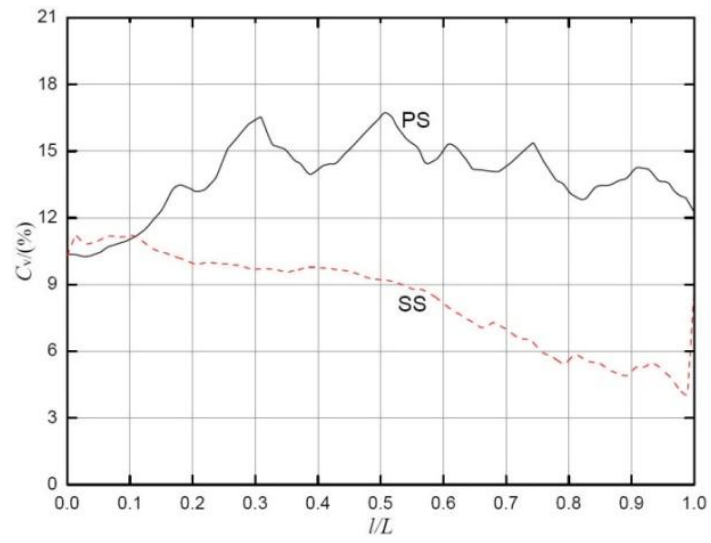

(a) 500

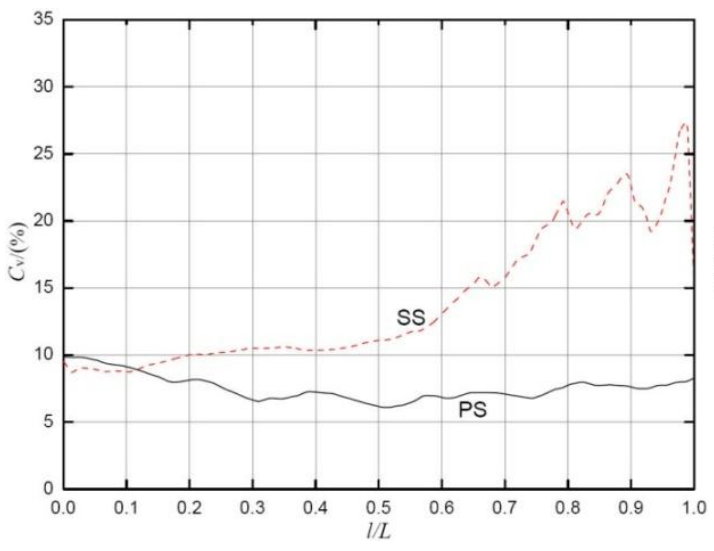

(b) 1500

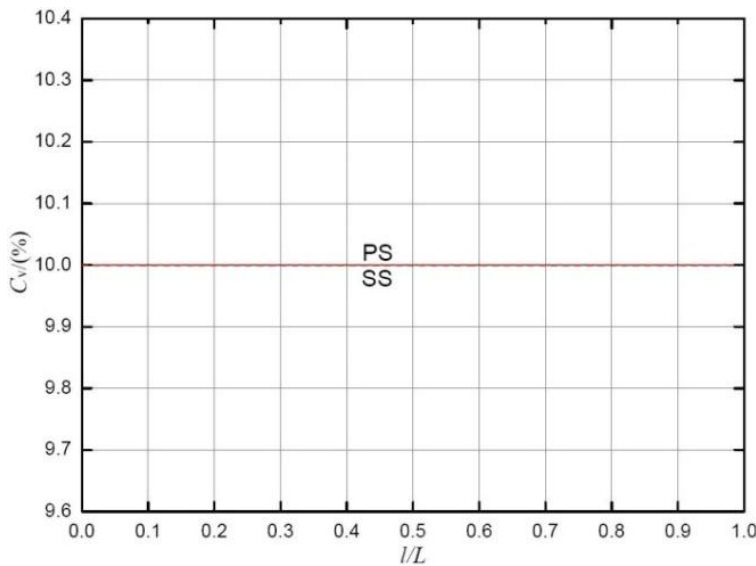

(b) 1000

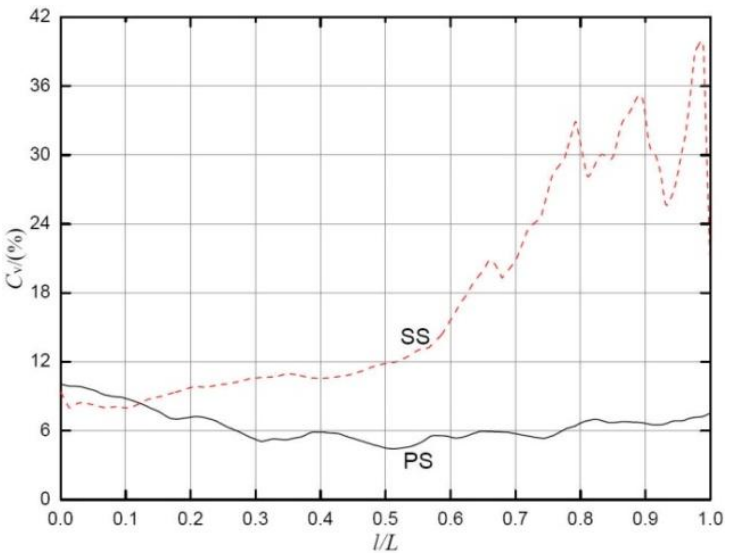

(d) 2000

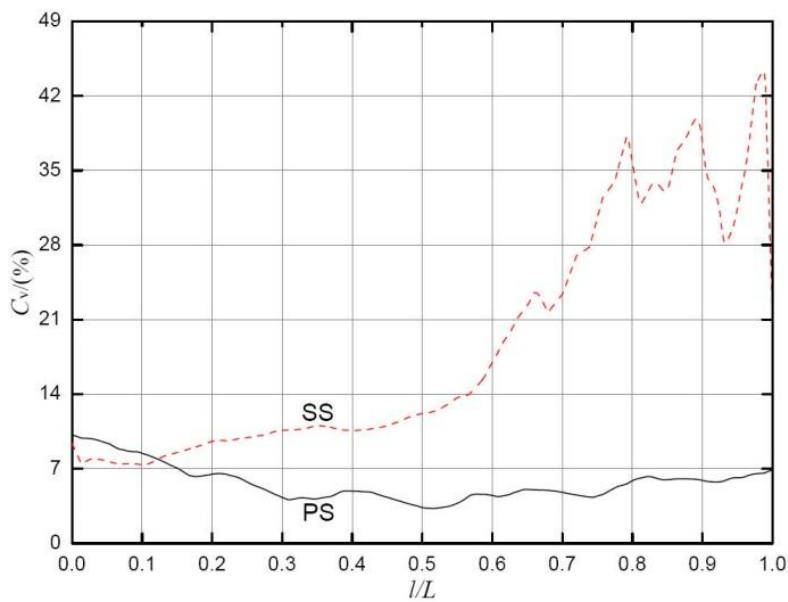

(e) 2500

Fig.3 Distribution of particle concentrations on the blade under different particle densities $\left(\mathrm{kg} / \mathrm{m}^{3}\right)$

\subsection{Total pressure and streamline}

Fig.4 shows total pressure and streamline obtained from the calculation under different densitiesof delivering solid-phase particles. Under different densitiesof delivering solid-phase particles, streamlines in all flow channels in the impeller were not greatly different and weregenerallyvery similar. The reason was that the density of delivering solid-phase particles was different, but the calculated working condition was the designed working condition, and the corresponding internal flow conditions at that time were generally good without a significantarea of stalled phenomenon. 


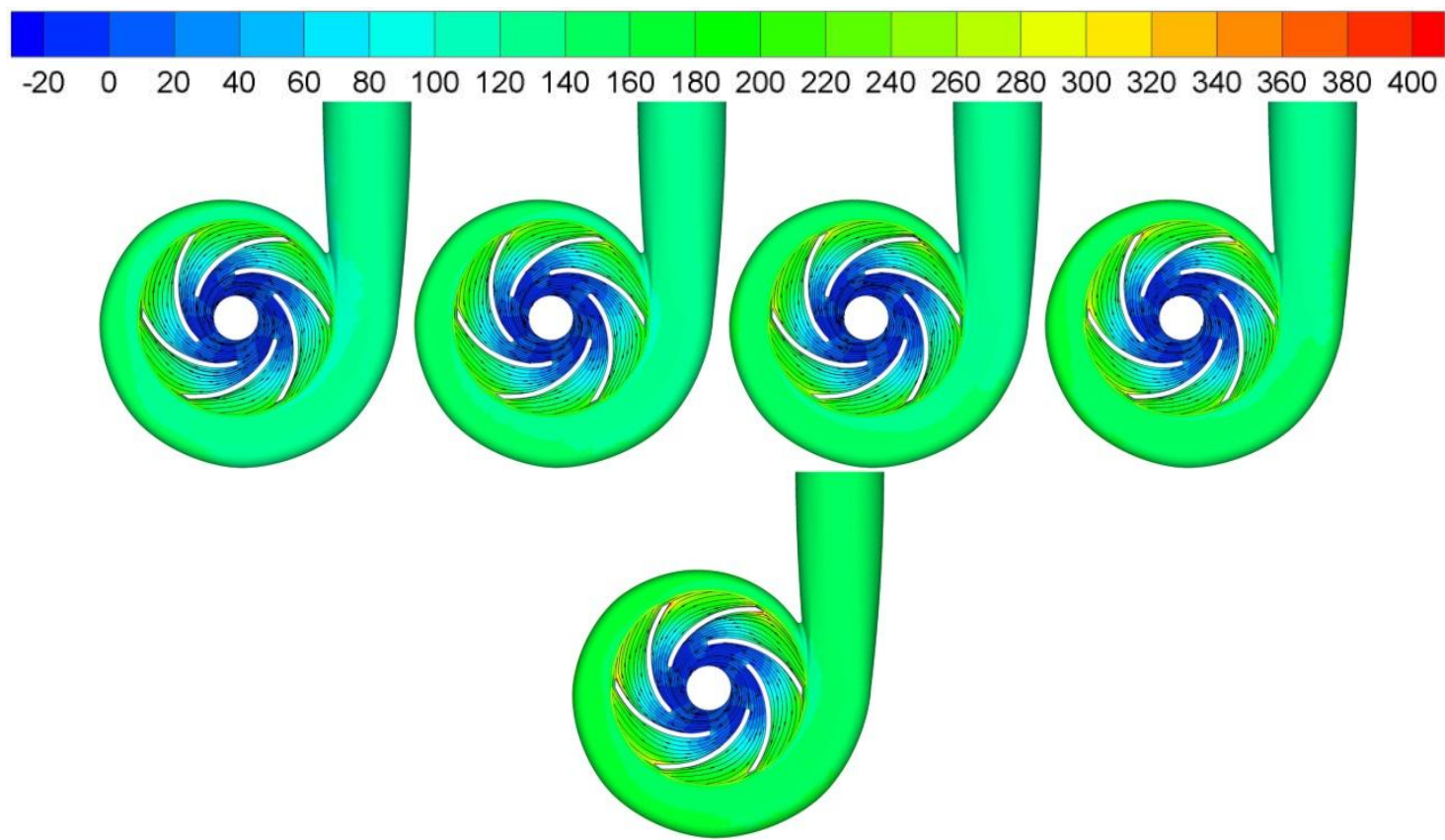
(a) 500
(b) 1000
(c) 1500
(d) 2000
(e) 2500

Fig.4Distribution of total pressure and streamline under different particle densities $\left(\mathrm{kg} / \mathrm{m}^{3}\right)$

\subsection{Difference in the external characteristics of solid-liquid two-phase flow}

Under the designed working condition $\left(Q=50 \mathrm{~m}^{3} / \mathrm{h}\right)$ and in the cases wherethe diameter of solid particle is $0.10 \mathrm{~mm}$, solid-phase delivering concentration is $10 \%$, and the density of solid particles is $2500 \mathrm{~kg} / \mathrm{m}^{3}$, the flow calculation of water and solid-liquid two-phase flow was carried out. The calculation result of the external characteristics of both is shown inFig.5. The calculation is carried out within the working condition of $0.60-1.40$ times that of rated flow. Under three working conditions that deliver flow at 30,50 , and $70 \mathrm{~m}^{3} / \mathrm{h}$, respectively, the calculation heads as delivering water medium are $22.03,20.68$, and $16.90 \mathrm{~m}$. When the calculation heads as delivering solid-liquid two-phase flow are $16.15,13.81$, and $7.61 \mathrm{~m}$, respectively, the differences under the corresponding flow between both of them are 5.88, 6.87, and $9.29 \mathrm{~m}$, respectively. Whether delivering a single-phase flow or a solid-liquid two-phase flow, hydraulic head was down with the increase of delivering flow. The hydraulic head delivering solid-liquid two-phase flow was obviously lower than that delivering water medium, and the difference between both increased with the increase of the flow rate.

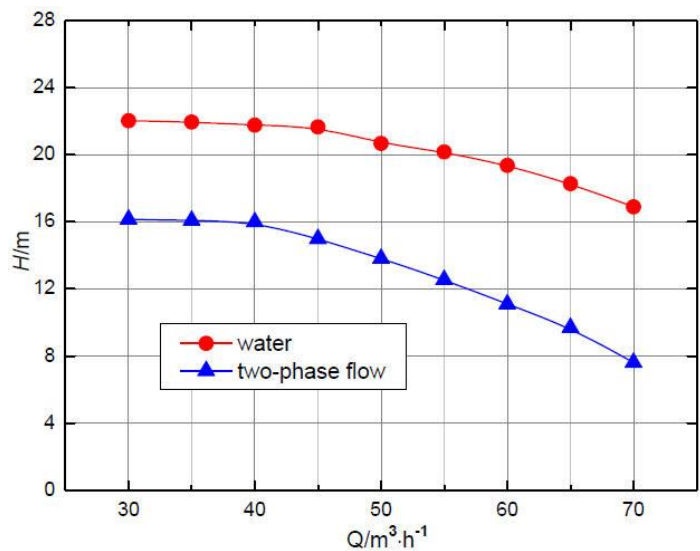

Fig.5 Comparison of external characteristics between delivering water and solid-liquid two-phase flow

\subsection{Solid-phase concentration distribution}

Under the same condition, solid-phase concentration distribution on the pump surface as the model pump was delivering solid-liquid two-phase flow under three working conditions (flow rates were 35,50 , and $65 \mathrm{~m}^{3} / \mathrm{h}$ ) is shown in Fig.6. The solid-liquid concentration distributions under three conditions were similar. This finding indicated that the degree of wear on theinternal surface of the volute, the front cover, and the back cover were similar. 


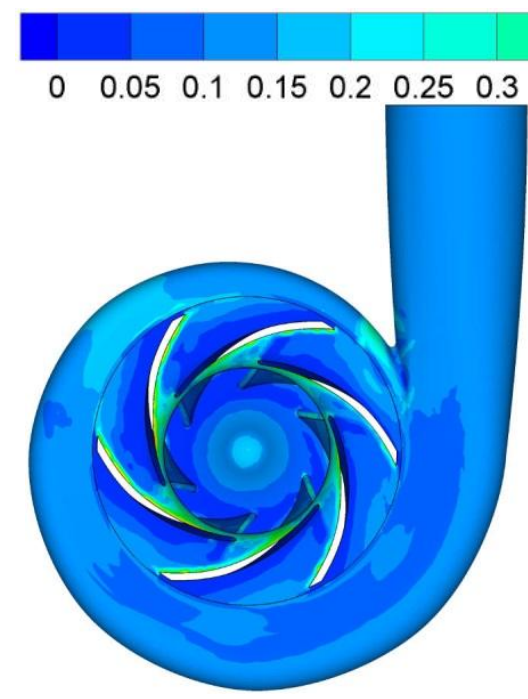

(a) 0.7

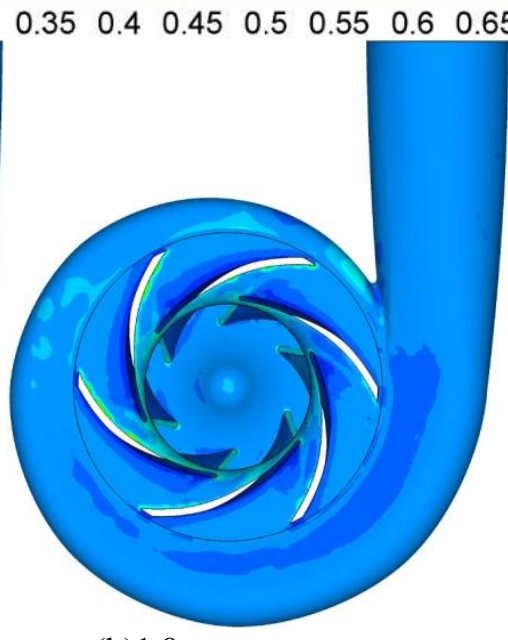

(b) 1.0

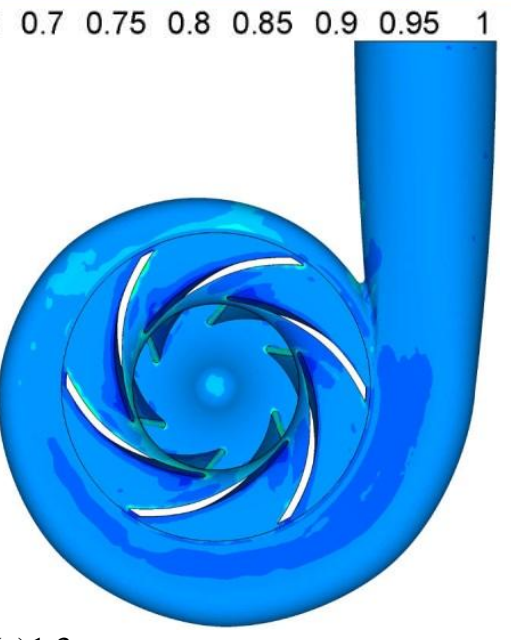

(c) 1.3

4 Conclusions

Fig.6Solid-phase concentration distributions on pump surface $\left(Q / Q_{\mathrm{d}}\right)$

(1) With the increase of delivering solid-phase density, the position of isobaric equilibrium point was moving to the front edge of the blade, but the position of solid-phase isoconcentration point on the blade pressure surface and suction surface was basically unchanged. This result indicated that the wear and tear on the blade pressure surface and suction surface was not changed with the change of particle density.

(2) Hydraulic head as delivering solid-liquid two-phase flow was significantly lower than water head, and the difference between them increased with the increase of flow rate.

\section{ACKNOWLEDGEMENT}

The work was supported by the Zhejiang Provincial Science and Technology Project (No.2015C31129, No.2016C31127), Academic Foundation of Quzhou University (No.XNZQN201508).

\section{REFERENCES}

[1]. Engin T, Gur M. Performance characteristics of centrifugal pump impeller with running tip clearance pumping solid-liquid mixtures[J]. Journal of Fluids Engineering, 2001, 123(3): 532-538.

[2]. Gandhi B K, Singh S N, Seshadn V. Effect of speed on the performance characteristics of a centrifugal slurry pump[J]. Journal of Hydraulic Engineering, 2002, 128(2): 225-233.

[3]. Yuan Shouqi, Zhang Peifang, Zhang Jinfeng. Numerical simulation of 3-D dense solid- liquid two- phase turbulent flow in a non-clogging mud pump[J]. Chinese Journal of Mechanical Engineering, 2004, 17(4):623-627.

[4]. Li Yi, Zhu Zuchao, He Weiqiang, et al. Numerical simulation and experimental research on the influence of solid phase characteristics on centrifugal pump performance[J]. Chinese Journal of Mechanical Engineering, 2012, 25(6): 1184-1189.

[5]. Zhang Yuliang, Li Yi, Cui Baoling, et al. Numerical simulation and analysis of solid-liquid two-phase flow in centrifugal pump[J]. Chinese Journal of Mechanical Engineering, 2013, 26(1): 53-60.

[6]. Zhang Yuliang, Li Yi, Zhu Zuchao, et al. Computational analysis of centrifugal pump delivering solid-liquid two-phase flow during startup period[J]. Chinese Journal of Mechanical Engineering, 2014, 27(1): 178-185.

[7]. Harry H T, Graeme R A. Experimental study on erosive wear of some metallic materials using Coriolis wear testing approach[J]. Wear, 2005, (258): 458-469.

[8]. Veselin B. Erosive wear model of slurry pump impeller[J]. Journal of Tribology, 2010, 132(2): 021602.1 5.

[9]. Pagalthivarthi K V, Gupta P K, Tyagi V, et al. CFD prediction of erosion wear in centrifugal slurry pumps for dilute slurry flows[J]. Journal of Computational Multiphase Flows, 2011, 3(4): 225-245.

[10]. Dong Xing, Zhang Hailu, Wang Xinyong. Finite element analysis of wear for centrifugal slurry pump[C]. The 6th International Conference on Mining Science \& Technology, 2009, (1): 1532-1538.

[11]. Li Yi, Zhu Zuchao, He Zhaohui, et a1. Abrasion characteristic analyses of solid-liquid two-phase centrifugal pump[J]. Journal of Thermal Science, 2010, 20(3): 283-287.

[12]. Sanyal J, Vasquez S, Roy S, et al. Numerical simulation of gas-liquid dynamics in cylindrical bubble column reactors [J]. Chemical Engineering Science, 1999, 54(21): 5071-5083. 